\title{
Formulation and solution to time-fractional generalized Korteweg-de Vries equation via variational methods
}

\section{Youwei Zhang ${ }^{*}$}

\section{"Correspondence:}

ywzhang0288@163.com

Department of Mathematics, Hexi

University, Beihuan Road 87,

Zhangye, Gansu 734000, China

\section{空 Springer}

\begin{abstract}
This paper presents the formulation of the time-fractional generalized Korteweg-de Vries equation (KdV) using the Euler-Lagrange variational technique in the Riemann-Liouville derivative sense and derives an approximate solitary wave solution. Our results show that He's variational-iteration method is a very efficient technique for finding the solution of the proposed equation and extend the existing results.
\end{abstract}

Keywords: Riemann-Liouville fractional differential operator; Euler-Lagrange equation; Riesz fractional derivative; generalized KdV equations; He's

variational-iteration method; solitary wave

\section{Introduction}

The KdV equation has been used to describe a wide range of physics phenomena of the evolution and interaction of nonlinear waves. It was derived from the propagation of dispersive shallow water waves and is widely used in fluid dynamics, aerodynamics, continuum mechanics, as a model for shock wave formation, solitons, turbulence, boundary layer behavior, mass transport, and the solution representing the water's free surface over a flat bed [1-3]. Camassa and Holm [4] put forward the derivation of solution as a model for dispersive shallow water waves and discovered that it is a formally integrable dimensional Hamiltonian system, and that its solitary waves are solitons. Most of the classical mechanics techniques have been used in studies of conservative systems, but most of the processes observed in the physical real world are nonconservative.

During the past three decades or so, fractional calculus has obtained considerable popularity and importance as generalization of integer-order evolution equations, and one has used it to model some meaningful things, such as fractional calculus in model price volatility in finance [5,6], in hydrology to model fast spreading of pollutants [7]; the most common hydrologic application of fractional calculus is the generation of fractional Brownian motion as a representation of aquifer material with a long-range correlation structure $[8,9]$. The fractional differential equation is used to model the particle motions in a heterogeneous environment and long particle jumps in anomalous diffusion in physics $[10,11]$. For other exact descriptions of the applications of engineering, mechanics, mathematics, etc., we can refer to [12-16]. If the Lagrangian of conservative system is constructed using fractional derivatives, the resulting equations of motion can be nonconservative. Therefore, in many cases, real physical processes could be modeled in a reli-

O2014 Zhang; licensee Springer. This is an Open Access article distributed under the terms of the Creative Commons Attribution License (http://creativecommons.org/licenses/by/2.0), which permits unrestricted use, distribution, and reproduction in any medium, provided the original work is properly cited. 
able manner using fractional-order differential equations rather than integer-order equations [17]. Based on the stochastic embedding theory, Cresson [18] defined the fractional embedding of differential operators and provided a fractional Euler-Lagrange equation for Lagrangian systems, then investigated a fractional Noether theorem and a fractional Hamiltonian formulation of fractional Lagrangian systems. Herzallah and Baleanu [19] presented the necessary and sufficient optimality conditions for the Euler-Lagrange fractional equations of fractional variational problems and made an effort in determining in which spaces the functional must exist. Malinowska [20] proposed the Euler-Lagrange equations for fractional variational problems with multiple integrals and proved the fractional Noether-type theorem for conservative and nonconservative generalized physical systems. Riewe [21, 22] formulated a version of the Euler-Lagrange equation for problems of calculus of variation with fractional derivatives. Wu and Baleanu [23] developed some new variational-iteration formulas to find approximate solutions of fractional differential equations and determined the Lagrange multiplier in a more accurate way. For generalized fractional Euler-Lagrange equations and a fractional-order Van der Pol-like oscillator, we can refer to the works by Odzijewicz [24, 25] and Attari et al. [26], respectively. For other the known results refer to Baleanu et al. [27] and Inokuti et al. [28]. In view of the fact that most physical phenomena may be considered as nonconservative, they can be described using fractional-order differential equations. Recently, several methods have been used to solve nonlinear fractional evolution equations using techniques of nonlinear analysis, such as the Adomian decomposition method [29], the homotopy analysis method [30, 31], the homotopy perturbation method [32,33] and the Laguerre spectral method [34-36]. It was mentioned that the variational-iteration method has been used successfully to solve different types of integer and fractional nonlinear evolution equations.

In the present paper, He's variational-iteration method [37-40] is applied to solve the time-fractional generalized $\mathrm{KdV}$ equation

$$
{ }_{0}^{R} D_{t}^{\alpha} u(x, t)+a u^{p}(x, t) u_{x}(x, t)+b u_{x x x}(x, t)=0
$$

where $a, b$ are constants, $u(x, t)$ is a field variable, the subscripts denote the partial differentiation of the function $u(x, t)$ with respect to the parameter $x$ and $t . x \in \Omega(\Omega \subseteq \mathbb{R})$ is a space coordinate in the propagation direction of the field and $t \in T\left(=\left[0, t_{0}\right]\left(t_{0}>0\right)\right)$ is the time, which occurs in different contexts in mathematical physics. $a, b$ are constant coefficients not equal to zero. The dissipative $u_{x x x}$ term provides damping at small scales, and the nonlinear term $u^{p} u_{x}(p>0)$ (which has the same form as that in the KdV or 1-dimensional Navier-Stokes equations) stabilizes by transferring energy between large and small scales. For $p=1$, we can refer to the known results of the time-fractional KdV equation: formulation and solution using variational methods $[41,42]$. For $p>0, p \neq 1$, there is little material on the formulation and solution to time-fractional $\mathrm{KdV}$ equation. Thus the present paper considers the formulation and solution to a time-fractional $\mathrm{KdV}$ equation as the index of the nonlinear term satisfies $p>0, p \neq 1 .{ }_{0}^{R} D_{t}^{\alpha}$ denotes the Riesz fractional derivative. Making use of the variational-iteration method, this work is devoted to a formulation of a time-fractional generalized $\mathrm{KdV}$ equation and derives an approximate solitary wave solution.

This paper is organized as follows: Section 2 states some background material from fractional calculus. Section 3 presents the principle of He's variational-iteration method. Section 4 is devoted to a description of the formulation of the time-fractional generalized 
KdV equation using the Euler-Lagrange variational technique and to derive an approximate solitary wave solution. Section 5 presents an analysis for the obtained graphs and figures and discusses the present work.

\section{Preliminaries}

We recall the necessary definitions for the fractional calculus (see [43-45]) which are used throughout the remaining sections of this paper.

Definition 1 A real multivariable function $f(x, t), t>0$ is said to be in the space $C_{\gamma}, \gamma \in \mathbb{R}$ with respect to $t$ if there exists a real number $r(>\gamma)$, such that $f(x, t)=t^{r} f_{1}(x, t)$, where $f_{1}(x, t) \in C(\Omega \times T)$. Obviously, $C_{\gamma} \subset C_{\delta}$ if $\delta \leq \gamma$.

Definition 2 The left-hand side Riemann-Liouville fractional integral of a function $f \in C_{\gamma}$ $(\gamma \geq-1)$ is defined by

$$
\begin{aligned}
& { }_{0} I_{t}^{\alpha} f(x, t)=\frac{1}{\Gamma(\alpha)} \int_{0}^{t} \frac{f(x, \tau)}{(t-\tau)^{1-\alpha}} d \tau, \quad \alpha>0, t \in T, \\
& { }_{0} I_{t}^{0} f(x, t)=f(x, t) .
\end{aligned}
$$

Definition 3 The Riemann-Liouville fractional derivatives of the order $n-1 \leq \alpha<n$ of a function $f \in C_{\gamma}(\gamma \geq-1)$ are defined as

$$
\begin{aligned}
& { }_{0} D_{t}^{\alpha} f(x, t)=\frac{1}{\Gamma(n-\alpha)} \frac{\partial^{n}}{\partial t^{n}} \int_{0}^{t} \frac{f(x, \tau)}{(t-\tau)^{\alpha+1-n}} d \tau, \\
& { }_{t} D_{t_{0}}^{\alpha} f(x, t)=\frac{1}{\Gamma(n-\alpha)} \frac{\partial^{n}}{\partial t^{n}} \int_{t}^{t_{0}} \frac{f(x, \tau)}{(\tau-t)^{\alpha+1-n}} d \tau, \quad t \in T .
\end{aligned}
$$

Lemma 1 The integration formula of Riemann-Liouville fractional derivative for the order $0<\alpha<1$

$$
\int_{T} f(x, t)_{0} D_{t}^{\alpha} g(x, t) d t=\int_{T} g(x, t)_{t} D_{t_{0}}^{\alpha} f(x, t) d t
$$

is valid under the assumptions that $f, g \in C(\Omega \times T)$ and that for arbitrary $x \in \Omega,{ }_{t} D_{t_{0}}^{\alpha} f$, ${ }_{0} D_{t}^{\alpha} g$ exist at every point $t \in T$ and are continuous in $t$.

Definition 4 The Riesz fractional integral of the order $n-1 \leq \alpha<n$ of a function $f \in C_{\gamma}$ $(\gamma \geq-1)$ is defined as

$$
{ }_{0}^{R} I_{t}^{\alpha} f(x, t)=\frac{1}{2}\left({ }_{0} I_{t}^{\alpha} f(x, t)+{ }_{t} I_{t_{0}}^{\alpha} f(x, t)\right)=\frac{1}{2 \Gamma(\alpha)} \int_{0}^{t_{0}}|t-\tau|^{\alpha-1} f(x, \tau) d \tau,
$$

where ${ }_{0} I_{t}^{\alpha}$ and ${ }_{t} I_{t_{0}}^{\alpha}$ are, respectively, the left- and right-hand side Riemann-Liouville fractional integral operators.

Definition 5 The Riesz fractional derivative of the order $n-1 \leq \alpha<n$ of a function $f \in C_{\gamma}$ $(\gamma \geq-1)$ is defined by

$$
\begin{aligned}
{ }_{0}^{R} D_{t}^{\alpha} f(x, t) & =\frac{1}{2}\left({ }_{0} D_{t}^{\alpha} f(x, t)+(-1)^{n}{ }_{t} D_{t_{0}}^{\alpha} f(x, t)\right) \\
& =\frac{1}{2 \Gamma(n-\alpha)} \frac{d^{n}}{d t^{n}} \int_{0}^{t_{0}}|t-\tau|^{n-\alpha-1} f(x, \tau) d \tau,
\end{aligned}
$$


where ${ }_{0} D_{t}^{\alpha}$ and ${ }_{t} D_{t_{0}}^{\alpha}$ are, respectively, the left- and right-hand side Riemann-Liouville fractional differential operators.

Lemma 2 Let $\alpha>0$ and $\beta>0$ be such that $n-1<\alpha<n, m-1<\beta<m$ and $\alpha+\beta<n$, and let $f \in L_{1}(\Omega \times T)$ and ${ }_{0} I_{t}^{m-\alpha} f \in A C^{m}(\Omega \times T)$. Then we have the following index rule:

$$
{ }_{0}^{R} D_{t}^{\alpha}\left({ }_{0}^{R} D_{t}^{\beta} f(x, t)\right)={ }_{0}^{R} D_{t}^{\alpha+\beta} f(x, t)-\sum_{i=1}^{m}{ }_{0}^{R} D_{t}^{\beta-i} f(x, t) \mid{ }_{t=0} \frac{t^{-\alpha-i}}{\Gamma(1-\alpha-i)} .
$$

Remark 1 One can express the Riesz fractional differential operator ${ }_{0}^{R} D_{t}^{\alpha-1}$ of the order $0<\alpha<1$ as the Riesz fractional integral operator ${ }_{0}^{R} I_{\tau}^{1-\alpha}$, i.e.

$$
{ }_{0}^{R} D_{t}^{\alpha-1} f(x, t)={ }_{0}^{R} I_{t}^{1-\alpha} f(x, t), \quad t \in T .
$$

\section{Variational-iteration method}

The variational-iteration method provides an effective procedure for explicit and solitary wave solutions of a wide and general class of differential systems representing real physical problems. Moreover, the variational-iteration method can overcome the foregoing restrictions and limitations of approximate techniques so that it provides us with a possibility to analyze strongly nonlinear evolution equations. Therefore, we extend this method to solve the time-fractional $\mathrm{KdV}$ equation. The basic features of the variational-iteration method are outlined as follows.

We consider a nonlinear evolution equation that consists of a linear part $\mathcal{L} u(x, t)$, a nonlinear part $\mathcal{N} u(x, t)$, and a free term $g(x, t)$ represented as

$$
\mathcal{L} u(x, t)+\mathcal{N} u(x, t)=g(x, t) .
$$

According to the variational-iteration method, the $(n+1)$ th approximation solution of $(1)$ can be read using an iteration correction functional as

$$
u_{n+1}(x, t)=u_{n}(x, t)+\int_{0}^{t} \lambda(\tau)(\mathcal{L} \tilde{u}(x, \tau)+\mathcal{N} \tilde{u}(x, \tau)-g(x, \tau)) d \tau,
$$

where $\lambda(\tau)$ is a Lagrangian multiplier and $\tilde{u}(x, t)$ is considered as a restricted variation function, i.e., $\delta \tilde{u}(x, t)=0$. Extremizing the variation of the correction functional (2) leads to the Lagrangian multiplier $\lambda(\tau)$. The initial iteration $u_{0}(x, t)$ can be used as the initial value $u(x, 0)$. As $n$ tends to infinity, the iteration leads to the solitary wave solution of (1), i.e.

$$
u(x, t)=\lim _{n \rightarrow \infty} u_{n}(x, t)
$$

\section{Time-fractional generalized $\mathrm{KdV}$ equation}

The generalized $\mathrm{KdV}$ equation in $(1+1)$ dimensions is given by

$$
u_{t}(x, t)+a u^{p}(x, t) u_{x}(x, t)+b u_{x x x}(x, t)=0,
$$

where $p>0, a, b$ are constants, $u(x, t)$ is a field variable, $x \in \Omega$ is a space coordinate in the propagation direction of the field and $t \in T$ is the time. Employing a potential function 
$v(x, t)$ on the field variable, setting $u(x, t)=v_{x}(x, t)$, yields the potential equation of the generalized KdV equation (3) in the form

$$
v_{x t}(x, t)+a v_{x}^{p}(x, t) v_{x x}(x, t)+b v_{x x x x}(x, t)=0 .
$$

The Lagrangian of this generalized KdV equation (3) can be defined using the semiinverse method $[46,47]$ as follows. The functional of the potential equation (4) can be represented as

$$
J(v)=\int_{\Omega} d x \int_{T}\left(v(x, t)\left(c_{1} v_{x t}(x, t)+c_{2} a v_{x}^{p}(x, t) v_{x x}(x, t)+c_{3} b v_{x x x x}(x, t)\right)\right) d t,
$$

with $c_{i}(i=1,2,3)$ being unknown constants to be determined later. Integrating (5) by parts and taking $\left.v_{t}\right|_{\Omega}=\left.v_{x}\right|_{\Omega}=\left.v_{x}\right|_{T}=0$ yield

$$
\begin{aligned}
J(v)= & \int_{\Omega} d x \int_{T}\left(-c_{1} v_{x}(x, t) v_{t}(x, t)-\frac{c_{2} a}{p+1} v_{x}^{p+2}(x, t)\right. \\
& \left.-c_{3} b v_{x}(x, t) v_{x x x}(x, t)\right) d t .
\end{aligned}
$$

The constants $c_{i}(i=1,2, \ldots, 6)$ can be determined taking the variation of the functional (6) to make it optimal. By applying the variation of the functional, integrating each term by parts, and making use of the variation optimum condition of the functional $J(v)$, it yields the following expression:

$$
-2 c_{1} v_{x t}(x, t)-c_{2} a(p+2) v_{x}^{p}(x, t) v_{x x}(x, t)-2 c_{3} b v_{x x x x}(x, t)=0 .
$$

We notice that the obtained result (7) is equivalent to (4), so one sees that the constants $c_{i}(i=1,2, \ldots, 6)$ are, respectively,

$$
c_{1}=-\frac{1}{2}, \quad c_{2}=-\frac{1}{p+2}, \quad c_{3}=-\frac{1}{2} .
$$

In addition, the functional expression given by (6) obtains directly the Lagrangian form of the generalized $\mathrm{KdV}$ equation,

$$
\begin{aligned}
L\left(v_{t}, v_{x}, v_{x x x}\right)= & \frac{1}{2} v_{x}(x, t) v_{t}(x, t)+\frac{a}{(p+1)(p+2)} v_{x}^{p+2}(x, t) \\
& +\frac{b}{2} v_{x}(x, t) v_{x x x}(x, t) .
\end{aligned}
$$

Similarly, the Lagrangian of the time-fractional version of the generalized KdV equation could be read as

$$
\begin{aligned}
F\left({ }_{0} D_{t}^{\alpha} v, v_{x}, v_{x x x}\right)= & \frac{1}{2} v_{x}(x, t){ }_{0} D_{t}^{\alpha} v(x, t)+\frac{a}{(p+1)(p+2)} v_{x}^{p+2}(x, t) \\
& \left.\left.+\frac{b}{2} v_{x}(x, t) v_{x x x}(x, t), \quad \alpha \in\right] 0,1\right] .
\end{aligned}
$$


Then the functional of the time-fractional generalized KdV equation will take the form of the expression

$$
J(v)=\int_{\Omega} d x \int_{T} F\left({ }_{0} D_{t}^{\alpha} \nu, v_{x}, v_{x x x}\right) d t,
$$

where the time-fractional Lagrangian $F\left({ }_{0} D_{t}^{\alpha} v, v_{x}, v_{x x x}\right)$ is given by (8). Following Agrawal's method [48-50], the variation of the functional (9) with respect to $v(x, t)$ leads to

$$
\begin{aligned}
\delta J(v)= & \int_{\Omega} d x \int_{T}\left(\frac{\partial F}{\partial_{0} D_{t}^{\alpha} \nu} \delta\left({ }_{0} D_{t}^{\alpha} v(x, t)\right)+\frac{\partial F}{\partial v_{x}} \delta v_{x}(x, t)\right. \\
& \left.+\frac{\partial F}{\partial v_{x x x}} \delta v_{x x x}(x, t)\right) d t .
\end{aligned}
$$

By Lemma 1, upon integrating the right-hand side of (10), one has

$$
\delta J(v)=\int_{\Omega} d x \int_{T}\left({ }_{t} D_{T}^{\alpha}\left(\frac{\partial F}{\partial_{0} D_{t}^{\alpha} v}\right)-\frac{\partial}{\partial x}\left(\frac{\partial F}{\partial v_{x}}\right)-\frac{\partial^{3}}{\partial x^{3}}\left(\frac{\partial F}{\partial v_{x x x}}\right)\right) \delta v d t,
$$

noting that $\left.\delta v\right|_{T}=\left.\delta v\right|_{\Omega}=\left.\delta v_{x}\right|_{\Omega}=0$.

Obviously, optimizing the variation of the functional $J(v)$, i.e., $\delta J(v)=0$, yields the EulerLagrange equation for time-fractional generalized $\mathrm{KdV}$ equation as in the following expression:

$$
{ }_{t} D_{T}^{\alpha}\left(\frac{\partial F}{\partial_{0} D_{t}^{\alpha} v}\right)-\frac{\partial}{\partial x}\left(\frac{\partial F}{\partial v_{x}}\right)-\frac{\partial^{3}}{\partial x^{3}}\left(\frac{\partial F}{\partial v_{x x x}}\right)=0 .
$$

Substituting the Lagrangian of the time-fractional generalized KdV equation (8) into the Euler-Lagrange formula (11) one obtains

$$
{ }_{t} D_{T}^{\alpha}\left(\frac{1}{2} v_{x}(x, t)\right)-{ }_{0} D_{t}^{\alpha}\left(\frac{1}{2} v_{x}(x, t)\right)-a v_{x}^{p}(x, t) v_{x x}(x, t)-b v_{x x x x}(x, t)=0 .
$$

Once again, substituting the potential function $v_{x}(x, t)$ for $u(x, t)$ yields the time-fractional generalized $\mathrm{KdV}$ equation for the state function $u(x, t)$,

$$
\frac{1}{2}\left({ }_{0} D_{t}^{\alpha} u(x, t)-{ }_{t} D_{T}^{\alpha} u(x, t)\right)+a u^{p}(x, t) u_{x}(x, t)+b u_{x x x}(x, t)=0 .
$$

According to the Riesz fractional derivative ${ }_{0}^{R} D_{t}^{\alpha} u(x, t)$, the time-fractional generalized $\mathrm{KdV}$ equation represented in (12) can be written as

$$
{ }_{0}^{R} D_{t}^{\alpha} u(x, t)+a u^{p}(x, t) u_{x}(x, t)+b u_{x x x}(x, t)=0 .
$$

Acting from the left-hand side by the Riesz fractional operator ${ }_{0}^{R} D_{t}^{1-\alpha}$ on (13) leads to

$$
\begin{aligned}
& \frac{\partial}{\partial t} u(x, t)-\left.{ }_{0}^{R} D_{t}^{\alpha-1} u(x, t)\right|_{t=0} \frac{t^{\alpha-2}}{\Gamma(\alpha-1)} \\
& \quad+{ }_{0}^{R} D_{t}^{1-\alpha}\left(a u^{p}(x, t) \frac{\partial}{\partial x} u(x, t)+b \frac{\partial^{3}}{\partial x^{3}} u(x, t)\right)=0,
\end{aligned}
$$


from Lemma 2. In view of the variational-iteration method, combining with (14), the ( $n+$ 1)th approximate solution of (13) can be read using the iteration correction functional as

$$
\begin{aligned}
u_{n+1}(x, t)= & u_{n}(x, t)+\int_{0}^{t} \lambda(\tau)\left[\frac{\partial}{\partial \tau} u_{n}(x, \tau)-\left.{ }_{0}^{R} D_{\tau}^{\alpha-1} u_{n}(x, \tau)\right|_{\tau=0} \frac{\tau^{\alpha-2}}{\Gamma(\alpha-1)}\right. \\
& \left.+{ }_{0}^{R} D_{\tau}^{1-\alpha}\left(a \tilde{u}_{n}^{p}(x, \tau) \frac{\partial}{\partial x} \tilde{u}_{n}(x, \tau)+b \frac{\partial^{3}}{\partial x^{3}} \tilde{u}_{n}(x, \tau)\right)\right] d \tau, \quad n \geq 0,
\end{aligned}
$$

where the function $\tilde{u}_{n}(x, t)$ is considered as a restricted variation function, i.e., $\delta \tilde{u}_{n}(x, t)=0$. The extremum of the variation of (15) subject to the restricted variation function straightforwardly yields

$$
\begin{aligned}
\delta u_{n+1}(x, t) & =\delta u_{n}(x, t)+\int_{0}^{t} \lambda(\tau) \delta \frac{\partial}{\partial \tau} u_{n}(x, \tau) d \tau \\
& =\delta u_{n}(x, t)+\left.\lambda(\tau) \delta u_{n}(x, \tau)\right|_{\tau=t}-\int_{0}^{t} \frac{\partial}{\partial \tau} \lambda(\tau) \delta u_{n}(x, \tau) d \tau \\
& =0 .
\end{aligned}
$$

This expression reduces to the following stationary conditions:

$$
\frac{\partial}{\partial \tau} \lambda(\tau)=0, \quad 1+\lambda(\tau)=0,
$$

which are converted to the Lagrangian multiplier at $\lambda(\tau)=-1$. Therefore, the correction functional (15) takes the following form:

$$
\begin{aligned}
u_{n+1}(x, t)= & u_{n}(x, t)-\int_{0}^{t}\left[\frac{\partial}{\partial \tau} u_{n}(x, \tau)-\left.{ }_{0}^{R} I_{\tau}^{1-\alpha} u_{n}(x, \tau)\right|_{\tau=0} \frac{\tau^{\alpha-2}}{\Gamma(\alpha-1)}\right. \\
& \left.+{ }_{0}^{R} D_{\tau}^{1-\alpha}\left(a u_{n}^{p}(x, \tau) \frac{\partial}{\partial x} u_{n}(x, \tau)+b \frac{\partial^{3}}{\partial x^{3}} u_{n}(x, \tau)\right)\right] d \tau, \quad n \geq 0,
\end{aligned}
$$

and since $\alpha-1<0$, the fractional derivative operator ${ }_{0}^{R} D_{t}^{\alpha-1}$ reduces to the fractional integral operator ${ }_{0}^{R} I_{t}^{1-\alpha}$ by Remark 1 .

The right-hand side Riemann-Liouville fractional derivative is interpreted as a future state of the process in physics. For this reason, the right-derivative is usually neglected in applications, when the present state of the process does not depend on the results of the future development, and so the right-derivative is used equal to zero in the following calculations. The zeroth order solitary wave solution can be taken as the initial value of the state variable, which is taken in this case as

$$
u_{0}(x, t)=u(x, 0)=k \operatorname{sech}^{\frac{2}{p}}\left(\frac{p}{2 \sqrt{b}}\left(x+\eta_{0}\right)\right)
$$

where $k=\left(\frac{(p+1)(p+2)}{2 a}\right)^{\frac{1}{p}}, \eta_{0}$ is a constant.

Substituting this zeroth order approximation solitary wave solution into (16) and using Definition 5 lead to the first order approximate solitary wave solution

$$
u_{1}(x, t)=k \operatorname{sech}^{\frac{2}{p}}\left(\frac{p}{2 \sqrt{b}}\left(x+\eta_{0}\right)\right)\left(1+\frac{t^{\alpha}}{\sqrt{b} \Gamma(\alpha+1)} \tanh \left(\frac{p}{2 \sqrt{b}}\left(x+\eta_{0}\right)\right)\right) .
$$


Substituting the first order approximate solitary wave solution into (16), using Definition 5 then leads to the second order approximate solitary wave solution in the following form:

$$
\begin{aligned}
& u_{2}(x, t)=k \operatorname{sech}^{\frac{2}{p}}\left(\frac{p}{2 \sqrt{b}}\left(x+\eta_{0}\right)\right) \\
& +\frac{t^{\alpha}}{\Gamma(\alpha+1)}\left[\frac{a k^{p+1}}{\sqrt{b}} \operatorname{sech}^{\frac{2}{p}+2}\left(\frac{p}{2 \sqrt{b}}\left(x+\eta_{0}\right)\right) \tanh \left(\frac{p}{2 \sqrt{b}}\left(x+\eta_{0}\right)\right)\right. \\
& +\frac{k}{\sqrt{b}} \operatorname{sech}^{\frac{2}{p}}\left(\frac{p}{2 \sqrt{b}}\left(x+\eta_{0}\right)\right) \tanh ^{3}\left(\frac{p}{2 \sqrt{b}}\left(x+\eta_{0}\right)\right) \\
& -\frac{3 k p}{2 \sqrt{b}} \operatorname{sech}^{\frac{2}{\bar{p}}+2}\left(\frac{p}{2 \sqrt{b}}\left(x+\eta_{0}\right)\right)-\frac{k p^{2}}{2 \sqrt{b}} \operatorname{sech}^{\frac{2}{p}+2}\left(\frac{p}{2 \sqrt{b}}\left(x+\eta_{0}\right)\right) \\
& \left.\times \tanh \left(\frac{p}{2 \sqrt{b}}\left(x+\eta_{0}\right)\right)\right] \\
& -\frac{t^{2 \alpha}}{\Gamma(2 \alpha+1)}\left[\frac{a k^{p+1}}{b} \operatorname{sech}^{\frac{2}{p}+2}\left(\frac{p}{2 \sqrt{b}}\left(x+\eta_{0}\right)\right)\right. \\
& \times\left(\frac{p}{2}-\frac{3 p+2}{2} \tanh ^{2}\left(\frac{p}{2 \sqrt{b}}\left(x+\eta_{0}\right)\right)\right) \\
& -\frac{3 k p^{2}(p+2)}{8 b} \operatorname{sech}^{\frac{2}{p}}\left(\frac{p}{2 \sqrt{b}}\left(x+\eta_{0}\right)\right) \tanh ^{2}\left(\frac{p}{2 \sqrt{b}}\left(x+\eta_{0}\right)\right) \\
& -\frac{3 k p^{2}(p+2)}{8 b} \operatorname{sech}^{\frac{2}{p}+2}\left(\frac{p}{2 \sqrt{b}}\left(x+\eta_{0}\right)\right) \\
& +\frac{3 k p(p+2)^{2}}{8 b} \operatorname{sech}^{\frac{2}{p}}\left(\frac{p}{2 \sqrt{b}}\left(x+\eta_{0}\right)\right) \tanh ^{2}\left(\frac{p}{2 \sqrt{b}}\left(x+\eta_{0}\right)\right) \\
& +\frac{k p^{3}}{8 b} \operatorname{sech}^{\frac{2}{p}}\left(\frac{p}{2 \sqrt{b}}\left(x+\eta_{0}\right)\right) \\
& -\frac{k(p+2)^{3}}{8 b} \operatorname{sech}^{\frac{2}{p}}\left(\frac{p}{2 \sqrt{b}}\left(x+\eta_{0}\right)\right) \tanh ^{4}\left(\frac{p}{2 \sqrt{b}}\left(x+\eta_{0}\right)\right) \\
& +\frac{3 k(p+2)^{2}}{8 b} \operatorname{sech}^{\frac{2}{p}+2}\left(\frac{p}{2 \sqrt{b}}\left(x+\eta_{0}\right)\right) \tanh ^{2}\left(\frac{p}{2 \sqrt{b}}\left(x+\eta_{0}\right)\right) \\
& \left.+\frac{k p^{2}(p+2)}{4 b} \operatorname{sech}^{\frac{2}{p}+2}\left(\frac{p}{2 \sqrt{b}}\left(x+\eta_{0}\right)\right) \tanh ^{2}\left(\frac{p}{2 \sqrt{b}}\left(x+\eta_{0}\right)\right)\right] \\
& -\frac{\Gamma(2 \alpha+1) t^{3 \alpha}}{\Gamma(3 \alpha+1)(\Gamma(\alpha+1))^{2}}\left[\frac{a p k^{p+1}}{2 b \sqrt{b}} \operatorname{sech}^{\frac{2}{p}+2}\left(\frac{p}{2 \sqrt{b}}\left(x+\eta_{0}\right)\right)\right. \\
& \left.\times \tanh \left(\frac{p}{2 \sqrt{b}}\left(x+\eta_{0}\right)\right)\left(p-\frac{p+2}{2} \tanh ^{2}\left(\frac{p}{2 \sqrt{b}}\left(x+\eta_{0}\right)\right)\right)\right] .
\end{aligned}
$$

Making use of Definition 5 and the Maple package or Mathematics, we obtain $u_{3}(x, t)$, $u_{4}(x, t)$ and so on, and substituting the $n-1$ order approximate solitary wave solution into (16) leads to the $n$ order approximate solitary wave solution. As $n$ tends to infinity, the iteration leads to the solitary wave solution of the time-fractional generalized $\mathrm{KdV}$ equation

$$
u(x, t)=k \operatorname{sech}^{\frac{2}{p}}\left(\frac{p}{2 \sqrt{b}}\left(x-t+\eta_{0}\right)\right) .
$$




\section{Discussion}

The target of the present work is the effect of the fractional-order derivative on the structure and propagation of the resulting solitary waves obtained from time-fractional generalized KdV equations. We derive the Lagrangian of the generalized KdV equations by the semi-inverse method, and then we take a similar form for the Lagrangian to the timefractional generalized $\mathrm{KdV}$ equations. Using the Euler-Lagrange variational technique, we continue our calculations until the third order iteration. Meanwhile, our approximate calculations are carried out on the solution of the time-fractional generalized KdV equations taking into account the values of the coefficients and some meaningful values namely, $\alpha=1, \frac{4}{5}, \frac{1}{2}$ and $\frac{1}{5}$. The solitary wave solution of time-fractional generalized KdV equations are obtained. In addition, a 3-dimensional representation of the solution $u(x, t)$ for the time-fractional generalized KdV equations with space $x$ and time $t$ for different values of the order $\alpha$ is presented, respectively. In Figure 1, the solution $u$ is still a single soliton wave solution for all values of the order $\alpha$. It shows that the balancing scenario between nonlinearity and dispersion is still valid. Figure 2 presents the change of amplitude and width of the soliton due to the variation of the order $\alpha, 2$ - and 3-dimensional graphs depicted the behavior of the solution $u(x, t)$ at time $t=1$ corresponding to different values of the order $\alpha$. This behavior indicates that the increasing of the value $\alpha$ is uniform in both the height and the width of the solitary wave solution. That is, the order $\alpha$ can be
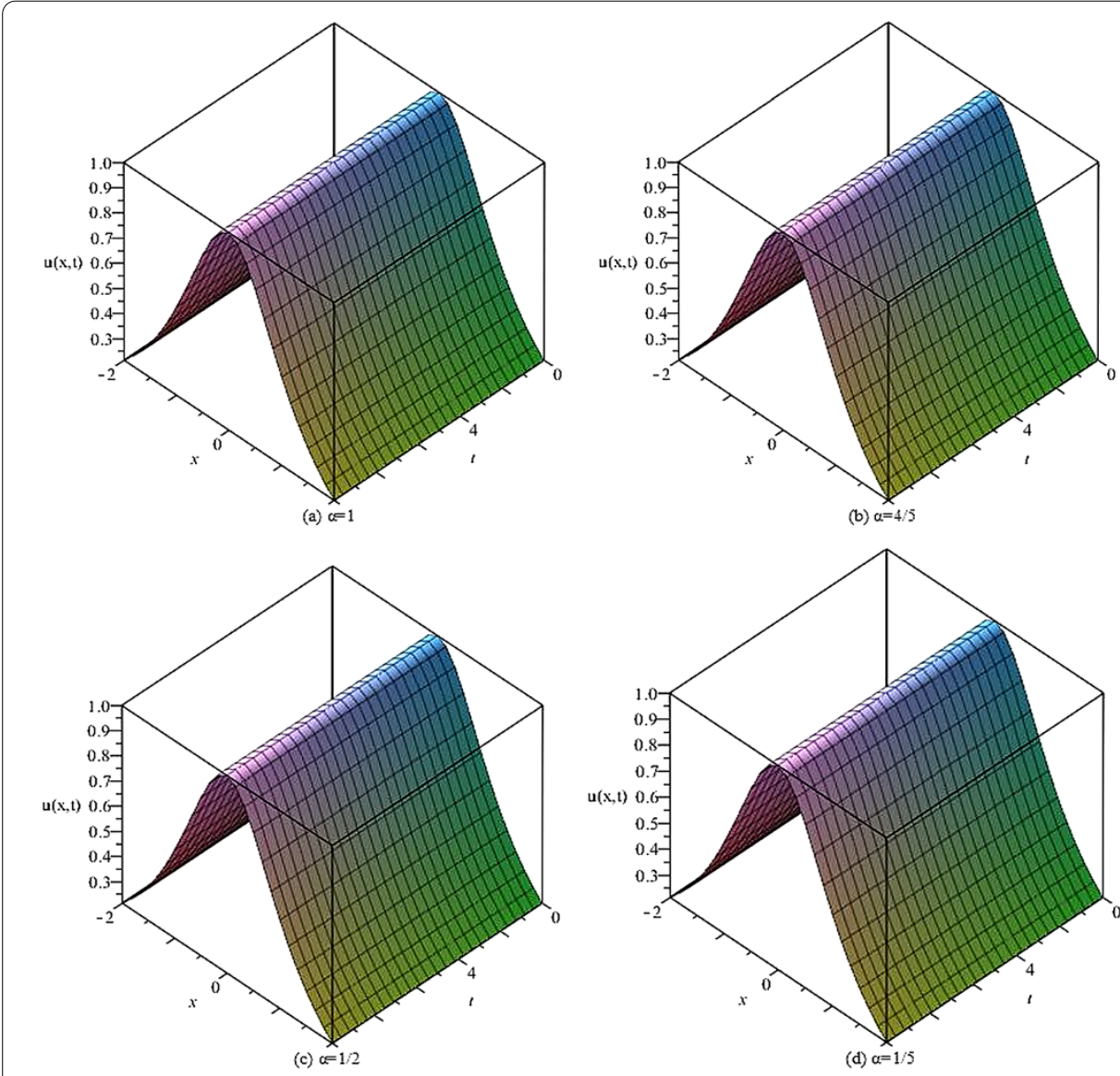

Figure 1 The function $u$ as a 3-dimensions graph for different order $\alpha$ when $p=3, a=10, b=1, \eta_{0}=0$. 

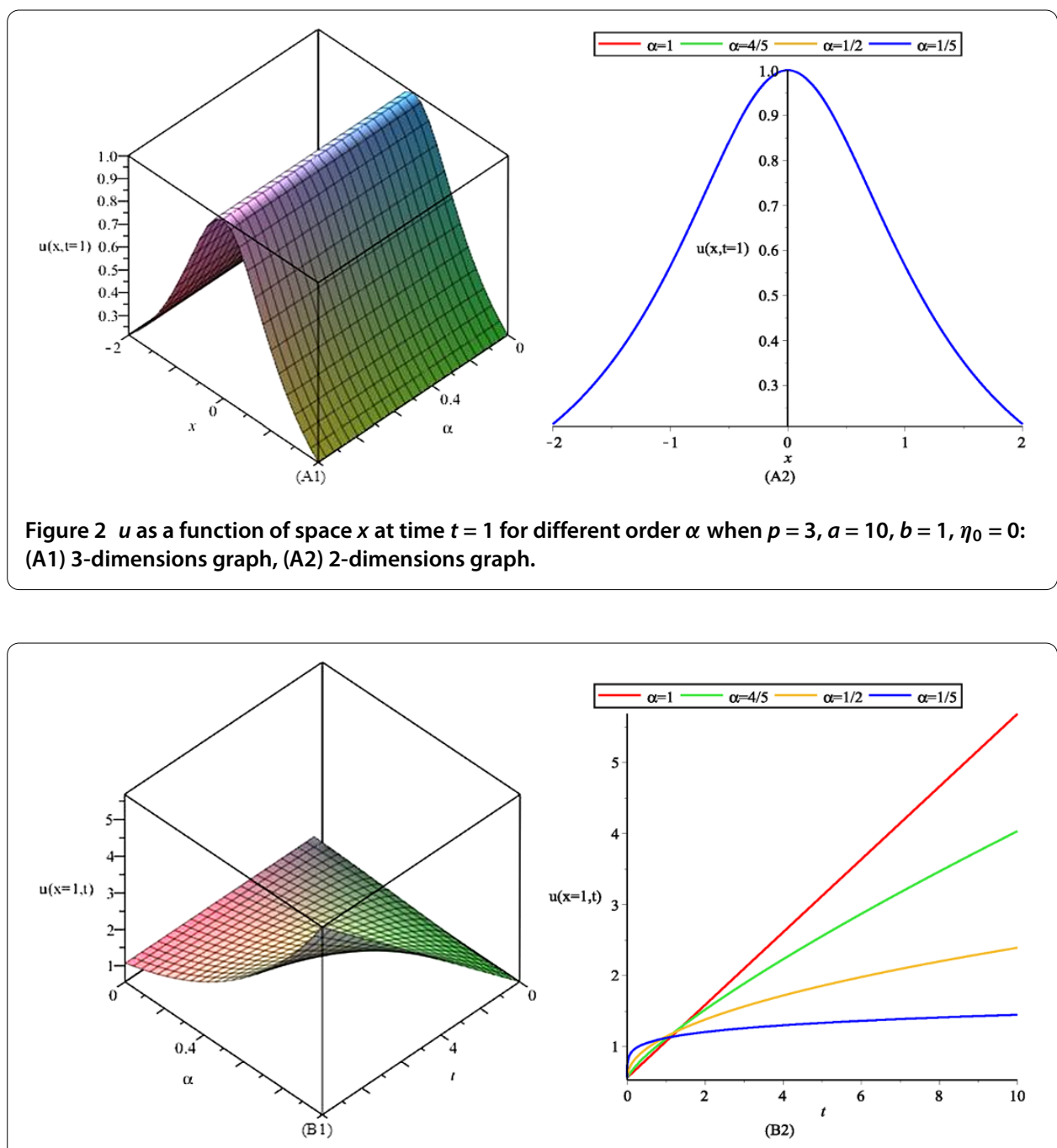

Figure 3 The amplitude of the function $u$ as a function of time $t$ at space $x=1$ of the fractional order $\alpha$ when $p=3, a=10, b=1, \eta_{0}=0$ : (B1) 3-dimensions graph, (B2) 2-dimensions graph.

used to modify the shape of the solitary wave without change of the nonlinearity and the dispersion effects in the medium. Figure 3 is devoted to the study of the expression of the relation between the amplitude of the soliton and the fractional order at different time values. These figures show that at the same time, the increasing of the fractional $\alpha$ increases the amplitude of the solitary wave to some value of $\alpha$.

Competing interests

The author declares that they have no competing interests.

\section{Author's contributions}

The author wrote this paper carefully, gave a rigorous derivation process, and read and approved the final manuscript.

Received: 7 November 2013 Accepted: 13 January 2014 Published: 14 Feb 2014

\section{References}

1. Camassa, R, Holm, D, Hyman, J: A new integrable shallow water equation. Adv. Appl. Mech. 31, 1-33 (1994)

2. Johnson, RS: Camassa-Holm, Korteweg-de Vries and related models for waterwaves. J. Fluid Mech. 455, 63-82 (2002)

3. Fokas, $A$, Fuchssteiner, B: Symplectic structures, their Bäcklund transformation and hereditary symmetries. Physica D 4, 47-66 (1981) 
4. Camassa, R, Holm, D: An integrable shallow water equation with peaked solutions. Phys. Rev. Lett. 71, 1661-1664 (1993)

5. Gorenflo, R, Mainardi, F, Scalas, E, Raberto, M: Fractional calculus and continuous-time finance, III. The diffusion limit. In: Mathematical Finance (Konstanz, 2000). Trends Math., pp. 171-180 (2001)

6. Sabatelli, L, Keating, S, Dudley, J, Richmond, P: Waiting time distributions in financial markets. Eur. Phys. J. B 27, 273-275 (2002)

7. Schumer, R, Benson, DA, Meerschaert, MM, Wheatcraft, SW: Eulerian derivation of the fractional advection-dispersion equation. J. Contam. Hydrol. 48, 69-88 (2001)

8. Benson, DA, Meerschaert, MM, Revielle, J: Fractional calculus in hydrologic modeling: a numerical perspective. Adv. Water Resour. 51, 479-497 (2013)

9. Roop, JP: Computational aspects of FEM approximation of fractional advection dispersion equations on bounded domains in $\mathbb{R}^{2}$. J. Comput. Appl. Math. 193, 243-268 (2006)

10. Hilfer, R: Applications of Fractional Calculus in Physics. World Scientific, Singapore (2000)

11. Meerschaert, MM, Benson, DA, Baeumer, B: Multidimensional advection and fractional dispersion. Phys. Rev. E 59, 5026-5028 (1999)

12. Lundstrom, B, Higgs, M, Spain, W, Fairhall, A: Fractional differentiation by neocortical pyramidal neurons. Nat. Neurosci. 11, 1335-1342 (2008)

13. Metzler, R, Klafter, J: Boundary value problems for fractional diffusion equations. Phys. A, Stat. Mech. Appl. 278, 107-125 (2000)

14. Rossikhin, YA, Shitikova, MV: Application of fractional derivatives to the analysis of damped vibrations of viscoelastic single mass systems. Acta Mech. 120, 109-125 (1997)

15. Schumer, R, Benson, DA, Meerschaert, MM, Baeumer, B: Multiscaling fractional advection-dispersion equations and their solutions. Water Resour. Res. 39, 1022-1032 (2003)

16. Yuste, $\mathrm{SB}, \mathrm{Acedo}, \mathrm{L}$, Lindenberg, $\mathrm{K}:$ Reaction front in an $\mathrm{A}+\mathrm{B} \rightarrow \mathrm{C}$ reaction subdiffusion process. Phys. Rev. E 69, $036126(2004)$

17. Tavazoei, MS, Haeri, M: Describing function based methods for predicting chaos in a class of fractional order differential equations. Nonlinear Dyn. 57, 363-373 (2009)

18. Cresson, J: Fractional embedding of differential operators and Lagrangian systems. J. Math. Phys. 48, 033504 (2007)

19. Herzallah, MAE, Baleanu, D: Fractional Euler-Lagrange equations revisited. Nonlinear Dyn. 69, 977-982 (2012)

20. Malinowska, AB: A formulation of the fractional Noether-type theorem for multidimensional Lagrangians. Appl. Math. Lett. 25, 1941-1946 (2012)

21. Riewe, F: Nonconservative Lagrangian and Hamiltonian mechanics. Phys. Rev. E 53, 1890-1899 (1996)

22. Riewe, F: Mechanics with fractional derivatives. Phys. Rev. E 55, 3581-3592 (1997)

23. Wu, GC, Baleanu, D: Variational iteration method for the Burgers' flow with fractional derivatives - new Lagrange multipliers. Appl. Math. Model. 37, 6183-6190 (2013)

24. Odzijewicz, T, Malinowska, AB, Torres, DFM: Generalized fractional calculus with applications to the calculus of variations. Comput. Math. Appl. 64, 3351-3366 (2012)

25. Odzijewicz, T, Malinowska, AB, Torres, DFM: Fractional variational calculus with classical and combined Caputo derivatives. Nonlinear Anal. TMA 75, 1507-1515 (2012)

26. Attari, M, Haeri, M, Tavazoei, MS: Analysis of a fractional order Van der Pol-like oscillator via describing function method. Nonlinear Dyn. 61, 265-274 (2010)

27. Baleanu, D, Muslih, Sl: Lagrangian formulation of classical fields within Riemann-Liouville fractional derivatives. Phys. Scr. $72,119-123(2005)$

28. Inokuti, M, Sekine, H, Mura, T: General use of the Lagrange multiplier in non-linear mathematical physics. In: Nemat-Nasser, S (ed.) Variational Method in the Mechanics of Solids. Pergamon, Oxford (1978)

29. Saha Ray, S, Bera, R: An approximate solution of a nonlinear fractional differential equation by Adomian decomposition method. Appl. Math. Comput. 167, 561-571 (2005)

30. Cang, J, Tan, $Y, X u, H, L i a o, S:$ Series solutions of nonlinear fractional Riccati differential equations. Chaos Solitons Fractals 40, 1-9 (2009)

31. Liao, S: The proposed homotopy analysis technique for the solution of nonlinear problems. PhD thesis, Shanghai Jiao Tong University (1992)

32. Sweilam, NH, Khader, MM, Al-Bar, RF: Numerical studies for a multi-order fractional differential equation. Phys. Lett. A 371, 26-33 (2007)

33. Golmankhaneh, AK, Golmankhaneh, AK, Baleanu, D: Homotopy perturbation method for solving a system of Schrodinger-Korteweg-de Vries equation. Rom. Rep. Phys. 63, 609-623 (2011)

34. Baleanu, D, Bhrawy, AH, Taha, TM: Two efficient generalized Laguerre spectral algorithms for fractional initial value problems. Abstr. Appl. Anal. 2013, Article ID 546502 (2013)

35. Baleanu, D, Bhrawy, AH, Taha, TM: A modified generalized Laguerre spectral method for fractional differential equations on the half line. Abstr. Appl. Anal. 2013, Article ID 413529 (2013)

36. Bhrawy, AH, Baleanu, D: A spectral Legendre-Gauss-Lobatto collocation method for a space-fractional advection diffusion equations with variable coefficients. Rep. Math. Phys. 72, 219-233 (2013)

37. He, J: A new approach to nonlinear partial differential equations. Commun. Nonlinear Sci. Numer. Simul. 2, 230-235 (1997)

38. He, J: Approximate analytical solution for seepage flow with fractional derivatives in porous media. Comput. Methods Appl. Mech. Eng. 167, 57-68 (1998)

39. Molliq, RY, Noorani, MSM, Hashim, l: Variational iteration method for fractional heat- and wave-like equations. Nonlinear Anal., Real World Appl. 10, 1854-1869 (2009)

40. Momani, S, Odibat, Z, Alawnah, A: Variational iteration method for solving the space- and time-fractional KdV equation. Numer. Methods Partial Differ. Equ. 24, 261-271 (2008)

41. El-Wakil, S, Abulwafa, E, Zahran, M, Mahmoud, A: Time-fractional KdV equation: formulation and solution using variational methods. Nonlinear Dyn. 65, 55-63 (2011)

42. Atangana, A, Secer, A: The time-fractional coupled-Korteweg-de-Vries equations. Abstr. Appl. Anal. 2013, Article ID $947986(2013)$ 
43. Kilbas, AA, Srivastava, HM, Trujillo, JJ: Theory and Applications of Fractional Differential Equations. North-Holland Mathematics Studies, vol. 204. Elsevier, Amsterdam (2006)

44. Podlubny, I: Fractional Differential Equations. Academic Press, San Diego (1999)

45. Samko, SG, Kilbas, AA, Marichev, Ol: Fractional Integrals and Derivatives: Theory and Applications. Gordon \& Breach, New York (1993)

46. He, J: Semi-inverse method of establishing generalized variational principles for fluid mechanics with emphasis on turbo-machinery aerodynamics. Int. J. Turbo Jet-Engines 14, 23-28 (1997)

47. He, J: Variational iteration method - a kind of nonlinear analytical technique: some examples. Int. J. Non-Linear Mech. 34, 699-708 (1999)

48. Agrawal, OP: Formulation of Euler-Lagrange equations for fractional variational problems. J. Math. Anal. Appl. 272, 368-379 (2002)

49. Agrawal, OP: A general formulation and solution scheme for fractional optimal control problems. Nonlinear Dyn. 38, 323-337 (2004)

50. Agrawal, OP: Fractional variational calculus in terms of Riesz fractional derivatives. J. Phys. A, Math. Theor. 40, 62-87 (2007)

10.1186/1687-1847-2014-65

Cite this article as: Zhang: Formulation and solution to time-fractional generalized Korteweg-de Vries equation via variational methods. Advances in Difference Equations 2014, 2014:65

Submit your manuscript to a SpringerOpen ${ }^{\odot}$ journal and benefit from:

- Convenient online submission

- Rigorous peer review

Immediate publication on acceptance

- Open access: articles freely available online

- High visibility within the field

- Retaining the copyright to your article

Submit your next manuscript at $>$ springeropen.com 